\title{
The prolongation of pulse transit time after a stellate ganglion block: An objective indicator of a successful block
}

\author{
Young Uk Kim MD', Yuseon Cheong $\mathrm{MD}^{2}$, Yu Gyeong Kong $\mathrm{MD}^{3}$, Jonghyuk Lee $\mathrm{MD}^{3}$, \\ Sehun Kim $\mathrm{MD}^{3}$, Hong Gyu Choi $\mathrm{MD}^{1}$, Jeong Hun Suh $\mathrm{MD}^{3}$
}

YU Kim, Y Cheong, YG Kong, et al. The prolongation of pulse transit time after a stellate ganglion block: An objective indicator of a successful block. Pain Res Manag 2015;20(6):305-308.

BACKGROUND: An objective marker of successful stellate ganglion block (SGB) does not exist. Horner syndrome, which is currently used to determine the effect of SGB, is sometimes ambiguous.

OBJECTIVE: To investigate the change in pulse transit time (PTT) after SGB, and to evaluate the utility of PTT as an objective measure of successful SGB.

METHODS: Eight patients (34 to 62 years of age) underwent SGB for diagnosis or treatment of sympathetically mediated pain of the upper extremities. The success of the SGB was determined according to the presence of Horner syndrome. Electrocardiography, noninvasive blood pressure measurements and pulse oximetry were used to monitor all patients. PTT was measured using data saved on the WinDaq waveform browser.

RESULTS: PTT was measured at baseline and $3 \mathrm{~min}, 5 \mathrm{~min}$ and $10 \mathrm{~min}$ after the injection of a local anesthetic. At $3 \mathrm{~min}$ after SGB, the mean ( \pm SD) PTT was $624.6 \pm 20.5 \mathrm{~ms}$. At 5 min after injection, the mean PTT was $630.8 \pm 17.5 \mathrm{~ms}$. Prolonged PTT at $5 \mathrm{~min}$ was found to return to the baseline value at $10 \mathrm{~min}(613.6 \pm 14.7 \mathrm{~ms})$. According to the Friedman test, the differences from baseline values were significant $(\mathrm{P}=0.008)$.

CONCLUSION: Measurement of PTT at $5 \mathrm{~min}$ after local anesthetic injection can help to objectively determine the success of SGB.

Key Words: Horner syndrome; Objective measurement tool; Pulse transit time; Stellate ganglion block

tellate ganglion block (SGB) is a procedure used for the treatment $\checkmark$ and diagnosis of disorders mediated by the sympathetic nervous system. These disorders include complex regional pain syndrome, acute herpes zoster, postherpetic neuralgia, hyperhidrosis and vascular disease such as Raynaud's disease (1-6). The sympathetically maintained pain is diagnosed clinically by evaluating the analgesic effect of sympathetic block (SB) (7). SB leads to vascular relaxation, which results in improved blood flow. However, an objective marker of successful SGB does not exist, and Horner syndrome, which is used to determine the effect of SGB, is sometimes not clearly present. An objective way to identify increased blood flow in the upper extremity after SGB would be helpful. Pulse transit time (PTT) is defined as the time required for the arterial pulse pressure wave to travel from the aortic valve to the periphery, and can be used as an index of the increased blood flow resulting from the decrease in arterial resistance
La prolongation du temps de transit des impulsions après un bloc du ganglion stellaire : un indicateur objectif de la réussite de l'intervention

HISTORIQUE : Il n'y a pas de marqueur objectif de réussite du bloc du ganglion stellaire (BGS). Le syndrome de Horner, utilisé pour déterminer l'effet du BGS, est parfois ambigu.

OBJECTIF : Examiner le changement du temps de transit des impulsions (TTI) après un BGS et évaluer son utilité comme mesure objective de réussite de l'intervention.

MÉTHODOLOGIE : Huit patients de 34 à 62 ans ont subi un BGS pour diagnostiquer ou traiter une douleur à médiation sympathique des membres supérieurs. La réussite du BGS était déterminée en fonction de la présence du syndrome de Horner. Tous les patients étaient surveillés par électrocardiographie, mesures non invasives de la tension artérielle et saturométrie. Le TTI était mesuré au moyen de données sauvegardées dans le navigateur en forme d'onde WinDaq.

RÉSULTATS : Le TTI était mesuré avant, puis trois minutes, cinq minutes et dix minutes après l'injection d'un anesthésique local. Trois minutes après le BGS, l'augmentation moyenne du TTI était de 624,6 $\pm 20,5 \mathrm{~ms}$. Cinq minutes après l'injection, elle était de $630,8 \pm 17,5$ ms. Le TTI prolongé au bout de cinq minutes retrouvait la valeur de départ au bout de dix minutes $(613,6 \pm 14,7 \mathrm{~ms})$. D'après le test de Friedman, les différences étaient significatives par rapport aux valeurs de départ $(\mathrm{P}=0,008)$.

CONCLUSION : La mesure du TTI cinq minutes après l'injection locale d'anesthésique peut contribuer à déterminer la réussite objective du BGS.

after SB $(8,9)$. For this reason, we hypothesized that PTT measurements would be significantly associated with a successful block. The aim of the present pilot study was to investigate the change in PTT after SGB and to evaluate the usefulness of PTT as an objective measurement tool for successful SGB.

\section{METHODS}

The protocol for the present prospective pilot study was approved by the Institutional Review Board of the Asan Medical Center (Seoul, Republic of Korea) and was registered at the University of Ulsan (Seoul, Republic of Korea) under the number S2013-0866-0002. Patients between 34 and 62 years of age on whom SGB was performed for the diagnosis or treatment of sympathetically mediated neuropathic pain of the upper extremities were enrolled in the Pain Clinic at Asan Medical Center. All eligible patients were able to understand the study

${ }^{1}$ Department of Anesthesiology and Pain Medicine, Catholic Kwandong University of Korea College of Medicine, International St Mary's Hospital, Incheon; ${ }^{2}$ Department of Anesthesiology and Pain Medicine, Kangwon National University Hospital, Chuncheon; ${ }^{3}$ Department of Anesthesiology and Pain Medicine, Asan Medical Center, University of Ulsan College of Medicine, Seoul, Republic of Korea

Correspondence: Dr Jeong Hun Suh, Department of Anesthesiology and Pain Medicine, Asan Medical Center, University of Ulsan College of Medicine, Seoul, Republic of Korea, 86 Asanbyungwon-gil, Pungnap-dong, Songpa-gu, Seoul, Korea 138-736.

Telephone 82-2-3010-5630, fax 82-2-3010-6790, e-mail paindrsuh@gmail.com 


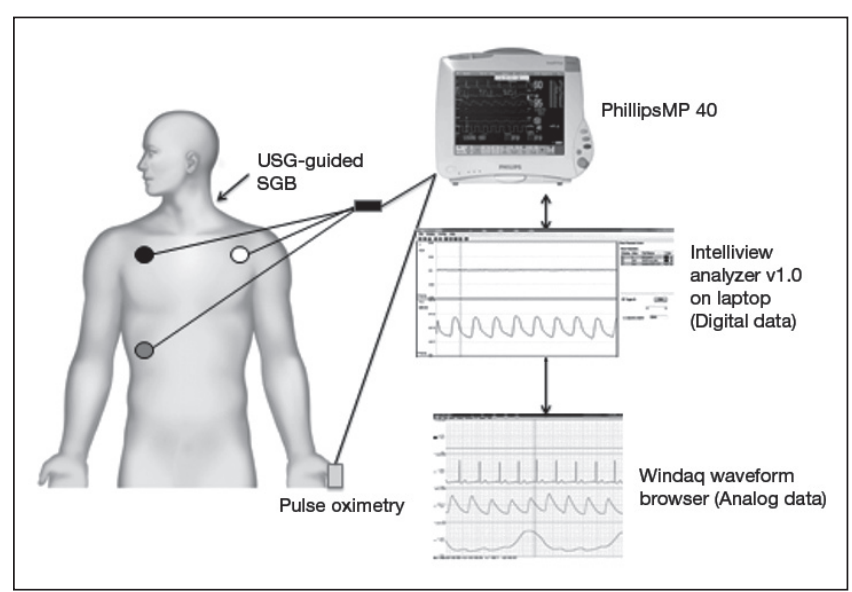

Figure 1) The pulse transit time monitoring equipment. Electrocardiography and a pulse oximeter were used in all patients. All monitoring values measured by a patient monitor (IntelliVue MP 60; Philips, Netherlands) were recorded automatically in real time on an interlocking computer program (version 1.0, Leomedics, Korea). Pulse transit time was measured using the saved data on the WinDaq ${ }^{\circledR}$ waveform browser. SGB Stellate ganglion block; USG Ultrasound

protocol, provide informed consent and participate in outcome measurements. Exclusion criteria were: diseases that can influence the vascular system such as vascular or valvular disease; use of antihypertensive medicine; and the presence of arrhythmia, diabetes or Raynaud's phenomenon. Patients with an essential tremor in the upper extremity or a history of adverse reactions to local anesthetics were also excluded. SGB was performed under ultrasound guidance by one pain specialist at Asan Medical Center. Although the stellate ganglion is located the C7-T1 vertebral level, SGB was performed at the C6 vertebral level because the vertebral artery is located in front of the anterior tubercle of C7. The cricoid cartilage is a landmark of the level of the C6 vertebra in the neutral supine position, and the target using the ultrasoundguided approach is the anterior tubercle of the C6 transverse process. The tip of the needle is located in the fascial plane where the stellate ganglion runs and is superficial to the fascia investing the longus colli muscle. Subsequently, $8 \mathrm{~mL}$ of $1 \%$ lidocaine was injected to produce a sympathetic block. After completion of the procedure, each patient was assessed for any signs of complications after the block. The sympatholytic effect after SGB was measured by examining for the presence of Horner syndrome (ptosis, miosis, red conjunctiva and facial anhidrosis). Electrocardiography (ECG), noninvasive blood pressure measurements and a pulse oximeter were used in all patients. All monitoring values measured by a patient monitor (IntelliVue MP 60; Philips, Netherlands) were recorded automatically in real time on an interlocking computer program (version 1.0, Leomedics, Korea). Monitoring began before performing the procedure and continued for $10 \mathrm{~min}$ after the injection of local anesthetics. The success of SGB was determined by confirming the presence of Horner syndrome. PTT was measured using saved data on the WinDaq waveform browser (DATAQ Instruments, USA). It was defined as the time between the appearance of the R wave on the ECG and the highest point of the corresponding wave on a plethysmogram, and was presented in milliseconds (Figure 1).

\section{Data analysis}

SPSS version 21 (IBM Corporation, USA) for Windows (Microsoft Corporation, USA) was used for data analysis; mean \pm SD and inferential statistics were obtained using the nonparametric Friedman test. $\mathrm{P}<0.05$ was considered to be statistically significant.

\section{RESULTS}

A total of eight patients (seven female, one male) were included. The patients had: a cervical herniated intervertebral disc with
TABLE 1

Patient characteristics

\begin{tabular}{lcclccc}
\hline Patient & \multicolumn{3}{c}{ Age, } & SGB & & $\begin{array}{c}\text { Horner } \\
\text { level }\end{array}$ \\
& Sex & years Diagnosis & C6 & Left & Yes \\
\hline 1 & Female & 55 & PHN & C6 & Right & Yes \\
2 & Female & 62 & C-HIVD with SMP & C6 & Right & Yes \\
3 & Male & 58 & PHN & C6 & Left & Yes \\
4 & Female & 55 & C-HIVD with SMP & C6 & Left & Yes \\
5 & Female & 52 & C-HIVD with SMP & C6 & Right & Yes \\
6 & Female & 56 & CRPS type I & C6 & Left & Yes \\
7 & Female & 49 & Central neuropathic pain & C6 & Right & Yes \\
8 & Female & 34 & CRPS type II with an \\
& & effect of brachial & C6 & & & \\
& & plexopathy & & & \\
\hline
\end{tabular}

CRPS Complex regional pain syndrome; C-HIVD Cervical herniated intervertebral disc; PHN Postherpetic neuralgia; SGB Stellate ganglion block; SMP Sympathetically maintained pain

sympathetically maintained pain (burning dysesthetic pain, swelling, hyperhidrosis) $(n=3)$; postherpetic neuralgia $(n=2)$; complex regional pain syndrome $(n=2)$; or central neuropathic pain $(n=1)$. The SGBs were successful in eight $(100 \%)$ cases (Table 1). An average PTT value was obtained, which was measured from five consequent waves. PTT was measured at baseline (PTT0) and 3 min (PTT3), $5 \mathrm{~min}$ (PTT5) and $10 \mathrm{~min}$ (PTT10) after the injection of lidocaine (Table 2).

At 3 min after SGB (PTT3), the mean PTT was $624.6 \pm 20.5 \mathrm{~ms}$. At 5 min after injection (PTT5), the mean PTT was $630.8 \pm 17.5 \mathrm{~ms}$. Prolonged PTT at $5 \mathrm{~min}$ returned to baseline at $10 \mathrm{~min}(613.6 \pm 14.7 \mathrm{~ms})$.

According to the Friedman test, the differences from baseline were significant $(\mathrm{P}=0.008)$ (Figure 2).

\section{DISCUSSION}

To our knowledge, changes in PTT after SGB have not previously been studied. We investigated the relationship between the change of PTT and the presence of clinical signs after SGB in patients with sympathetically mediated pain of the upper extremities to determine whether PTT could be used to verify the success of SGB. We found that changes in PTT can objectively predict a successful SGB after only $5 \mathrm{~min}$. This highly significant correlation was achieved with no complications. No patients dropped out of the present pilot study.

The stellate ganglion is part of the cervical sympathetic trunk and lies immediately anterior to the anterior tubercle of the $\mathrm{C} 7$ vertebrae. The sympathetic supply to the head, neck and upper limb is derived from the thoracic segments 1 to 9 and passes through the stellate ganglion $(1,3)$. An SGB is an injection of local anesthetic for pain located in the arm, chest, neck or head that is caused by sympathetically maintained pain resulting from reflex sympathetic dystrophy, postherpetic neuralgia, herpes zoster, causalgia or intractable angina (10). SGB also has an established use in treating patients with disorders mediated by circulatory insufficiency such as Raynaud's phenomenon or CREST syndrome. We performed a total of eight SGBs using our ultrasoundguided approach. The ultrasound-guided approach is a safe and valuable tool for confirming the spread of local anesthetics in the adequate fascial plane without risking exposure to radiation, and for guiding needle advancement (11). The stellate ganglion lies on the longus colli muscle at the C7-T1 vertebral level (12). Several studies have suggested that the local anesthetic solution would be discharged around and within the longus colli muscle during SGB (13). However, if the local anesthetic is not injected into the proper location, the sympatholytic effect cannot be identified in the upper limbs (6). We believe that the strength of PTT measurement can detect these misplaced blocks in the upper limb using a pulse oximeter sensor placed over the ipsilateral index finger. 
TABLE 2

\begin{tabular}{|c|c|c|c|c|}
\hline Patient & PTT0 & PTT3 & PTT5 & PTT10 \\
\hline 1 & 592 & 646.55 & 642 & 632 \\
\hline 2 & 616.8 & 612.7 & 624.3 & 619.6 \\
\hline 3 & 590.7 & 615.7 & 629 & 611 \\
\hline 4 & 559.7 & 598 & 603.3 & 591.3 \\
\hline 5 & 626.8 & 613.6 & 635.2 & 622.4 \\
\hline 6 & 655.6 & 658 & 664.4 & 610.8 \\
\hline 7 & 587.2 & 614 & 622.8 & 594.4 \\
\hline 8 & 606.8 & 638.4 & 625.6 & 627.2 \\
\hline
\end{tabular}

PTT values presented in milliseconds. PTTO PTT at baseline; PTT3 PTT 3 min after the injection of the local anesthetic; PTT5 PTT 5 min after the injection of the local anesthetic; PTT10 PTT 10 min after the injection of the local anesthetic

Ipsilateral Horner syndrome (facial anhidrosis, ptosis, miosis and red conjunctiva) is well known in patients after a successful SGB (14). However, Horner syndrome can only subjectively predict a successful block. Also, in some patients, these signs can be ambiguous for the evaluation of a successful block and there are no concrete defined criteria (6). In particular, it is known that the sympathetic function of the upper extremity is not blocked successfully by SGB, although the presence of clinical signs, such as anhidrosis, miosis, hypohidrosis, enophthalmos and bloodshot conjunctiva can be used as criteria $(7,15,16)$. Another conventional method for evaluating SGB is the change in temperature. Sympatholysis was measured in terms of the increase in temperature in the ipsilateral extremity after the block, and the increase in temperature was compared with that of the contralateral extremity (17). However, Krumova et al (7) reported that a skin temperature measurement is required to verify ipsilateral sympathicolysis at least $90 \mathrm{~min}$ after the intervention. To address the weak points of previous measurements, many recent clinical trials have been introduced to verify the efficacy of SGB. Yamazaki et al (6) reported that continuous measurements of the perfusion index using pulse oximetry could be useful in the determination of the efficacy of SGB. Murakawa et al (18) examined blood flow in the common carotid artery using an ultrasonic blood flow meter. They demonstrated that the common carotid artery blood flow increased after blocking SGB. Rogowski et al (19) reported that thermographic examination of the upper extremity on the ipsilateral side of stellate blockade is a useful diagnostic tool for the assessment of SGB. Compared with these measurement tools, PTT measurement is easy to use, inexpensive, and rapid and safe for assessing SGB objectively. To measure PTT, only three external ECG and photoplethysmographic sensors, which are basic and noninvasive monitoring devices, are required. Clinically, PTT changes can be used as a noninvasive beat-to-beat index of arterial pressure changes. Sympatholysis changes arterial distensibility via vasodilation and leads to a decrease of pulse wave velocity (PWV), which is a parameter of arterial distensibility $(20,21)$. PTT is inversely related to PWV (20). SB also decreases systemic vascular resistance (SVR). Thus, we assume that a lower SVR will result in lower tension of the arterial wall and a higher PTT, although further studies are required to examine these relationships. PWV can be determined by measuring the PTT of blood, whose flow fixes the distance of the blood vessel indirectly (20). Because of this, several studies have been performed. Kortekaas et al (22) reported that the mean PTT difference increased significantly after a successful axillary brachial plexus blockade. They concluded that PTT is a reliable method to evaluate whether a brachial plexus block is going to be successful. SharwoodSmith et al (21) found that PTT may be used to predict the onset of hypotension because PTT measurements give a beat-to-beat change of arterial pressure during spinal anesthesia in obstetric patients. In the

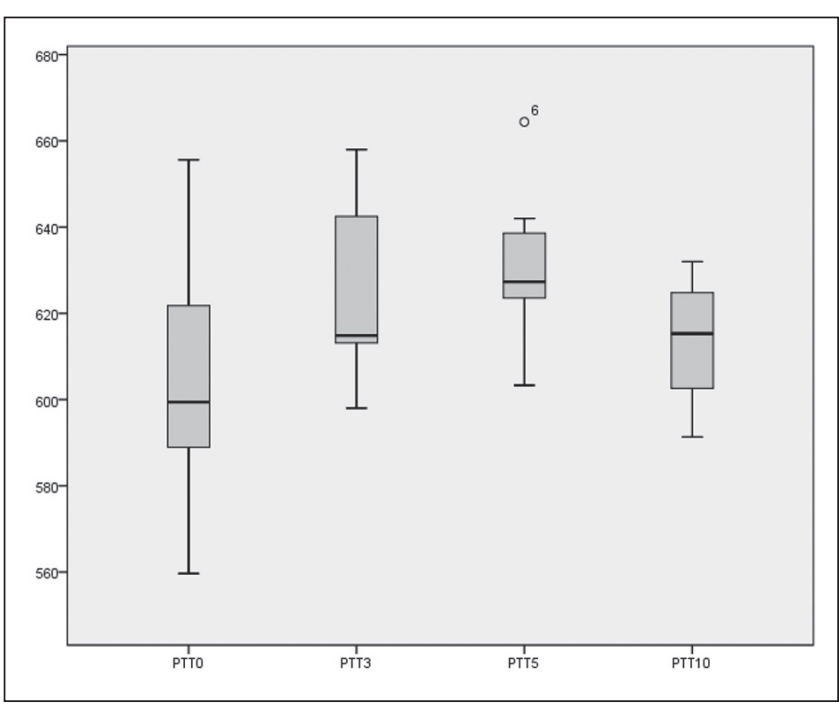

Figure 2) Box plot of data reflecting changes in mean pulse transit time (PTT) (ms) at baseline (PTT0) and $3 \mathrm{~min}$ (PTT3), 5 min (PTT5) and $10 \mathrm{~min}$ (PTT10) after the injection of the local anesthetic

present study, we investigated the success of SGB by performing PTT measurements on eight patients treated with SGB. All patients had Horner syndrome and PTT of the ipsilateral hand was prolonged at $5 \mathrm{~min}$ after SGB. This enabled us to predict successful SGB objectively. We also found that a prolonged PTT at 5 min returned to the baseline value at $10 \mathrm{~min}$. To our knowledge, there have been no previous reports regarding this phenomenon. We assume that the increased blood flow after SGB returns to normal via a redistribution of arterial blood flow, and it may take $10 \mathrm{~min}$. The PTT is a simplified surrogate marker to reflect blood flow, and may be affected by several mechanisms controlling the blood flow. We could not interpret this phenomenon exactly. However, we assumed that the change in PTT, not the value of PTT itself, reflect the change in blood flow and not the absolute value of blood flow. Therefore, the PTT increment was only shown during the time when the blood flow increased and then normalized because the blood flow maintained the increased state. Further investigation will be required to determine the time for redistribution.

There were several limitations to the present study. First, the number of patients was relatively small, suggesting the risk of an undersampling error. The results of our pilot study indicate a need for a larger, well-powered, properly conducted trial. The second limitation was that PTT may be affected by movement artifacts, which can cause artifacts in photoplethysmography signals. Fluctuations in the arousal state can lead to fluctuations in PTT and, thus, false scoring. The third limitation was that we did not compare the prolongation of the PTT with thermography in clinical trials, although thermography is a useful tool for the assessment of SGB effectiveness. In future studies, the correlation between PTT changes and thermographic changes should be elucidated in patients undergoing SGB. The fourth limitation was that we could not measure the response in the contralateral side as a control because our monitoring and recording system supported only one channel for the plethysmography. To overcome this point, we measured baseline PTT and considered this value as a control. The fifth limitation was that patients with Raynaud's phenomenon were excluded. Raynaud's phenomenon is associated with excessively reduced blood flow in response to emotional stress, causing discoloration of the fingers, and is believed to be the result of vasospasm that decreases blood supply to the extremities. Thus, we assumed Raynaud's phenomenon could affect experimental outcomes during PTT measurement. If possible, further investigations involving patients with Raynaud's phenomenon should be performed to evaluate the changes in PTT after SGB. 
Despite these limitations, the present study suggests that measurement of PTT at 5 min after local anesthetic injection can help to objectively determine whether SGB was successful.

\section{CONCLUSION}

To our knowledge, the present study was the first to measure the effect of SGB on PTT. The results of our study showed that the change in PTT during the $5 \mathrm{~min}$ after local anesthetic injection can help to determine whether SGB has been successful. This would allow PTT to be a potential measurement tool that is noninvasive, safe, easy to use and a rapid method of objectively assessing the success of SGB. However, comparisons with other tests, such as thermography, would be needed to support the clinical validity of these results.

\section{REFERENCES}

1. Guttuso T, Jr. Stellate ganglion block for treating hot flashes: A viable treatment option or sham procedure? Maturitas 2013;76:221-4.

2. Karnwal A, Liao W, Julka IS, Kakazu CZ. Stellate ganglion block for postoperative analgesia in patients with upper extremity orthopaedic injuries. Anaesthesia 2011;66:1052-3.

3. Kulkarni KR, Kadam AI, Namazi IJ. Efficacy of stellate ganglion block with an adjuvant ketamine for peripheral vascular disease of the upper limbs. Indian J Anaesth 2010;54:546-51.

4. Bhatia A, Flamer D, Peng PW. Evaluation of sonoanatomy relevant to performing stellate ganglion blocks using anterior and lateral simulated approaches: An observational study. Can J Anaesth 2012;59:1040-7.

5. Moon HS, Chon JY, Lee SH, et al. Long-term results of stellate ganglion block in patients with olfactory dysfunction. Korean J Pain 2013;26:57-61

6. Yamazaki H, Nishiyama J, Suzuki T. Use of perfusion index from pulse oximetry to determine efficacy of stellate ganglion block. Local Reg Anesth 2012;5:9-14.

7. Krumova EK, Gussone C, Regeniter S, Westermann A, Zenz M, Maier C. Are sympathetic blocks useful for diagnostic purposes? Reg Anesth Pain Med 2011;36:560-7.

8. Foo JY, Wilson SJ, Bradley AP. Physiologic parameters that affect pulse transit time difference between the upper and lower limbs in children. J Hum Hypertens 2006;20:221-3.

9. Babchenko A, Davidson E, Adler D, Ginosar Y, Kurz V, Nitzan M. Increase in pulse transit time to the foot after epidural anaesthesia treatment. Med Bio Eng 2000;38:674-9.
10. Park JS, Kim KJ, Lee YW, et al. Estimation of stellate ganglion block injection point using the cricoid cartilage as landmark through x-ray review. Korean J Pain 2011;24:141-5.

11. Ojeda A, Sala-Blanch X, Moreno LA, Busquets C. Ultrasoundguided stellate ganglion block: What about the phrenic nerve? Reg Anesth Pain Med 2013;38:170.

12. Elias M. Cervical sympathetic and stellate ganglion blocks. Pain Physician 2000;3:294-304.

13. Honma M, Murakami G, Sato TJ, Namiki A. Spread of injectate during C6 stellate ganglion block and fascial arrangement in the prevertebral region: An experimental study using donated cadavers. Reg Anesth Pain Med 2000;25:573-83.

14. van Gastel P, Kallewaard JW, van der Zanden M, de Boer H. Stellate-ganglion block as a treatment for severe postmenopausal flushing. Climacteric 2013;16:41-7.

15. Erickson SJ, Hogan QH. CT-guided injection of the stellate ganglion: Description of technique and efficacy of sympathetic blockade. Radiology 1993;188:707-9.

16. Schurmann M, Gradl G, Wizgal I, et al. Clinical and physiologic evaluation of stellate ganglion blockade for complex regional pain syndrome type I. Clin J Pain 2001;17:94-100.

17. Toshniwal G, Sunder R, Thomas R, Dureja GP. Management of complex regional pain syndrome type I in upper extremityevaluation of continuous stellate ganglion block and continuous infraclavicular brachial plexus block: A pilot study. Pain Med 2012;13:96-106.

18. Murakawa K, Ishimoto E, Noma K, Ishida K, Nishijima M, Izumi R. [Circulatory effects of stellate ganglion block in idiopathic facial palsy]. Masui 1994;43:356-60.

19. Rogowski J, Mrozinski P, Jagielak D, Lango R, Narkiewicz M, Wujtewicz M. Thermographic assessment of stellate ganglion block effectiveness during cardiosurgical procedures. Med Sci Monit 2000;6:407-10.

20. Nitzan M, Khanokh B, Slovik Y. The difference in pulse transit time to the toe and finger measured by photoplethysmography. Physiol Meas 2002;23:85-93.

21. Sharwood-Smith G, Bruce J, Drummond G. Assessment of pulse transit time to indicate cardiovascular changes during obstetric spinal anaesthesia. Br J Anaesth 2006;96:100-5.

22. Kortekaas MC, Niehof SP, van Velzen MH, Galvin EM, Huygen FJ, Stolker RJ. Pulse transit time as a quick predictor of a successful axillary brachial plexus block. Acta Anaesthesiol Scand 2012;56:1228-33. 


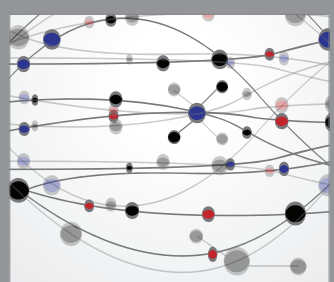

The Scientific World Journal
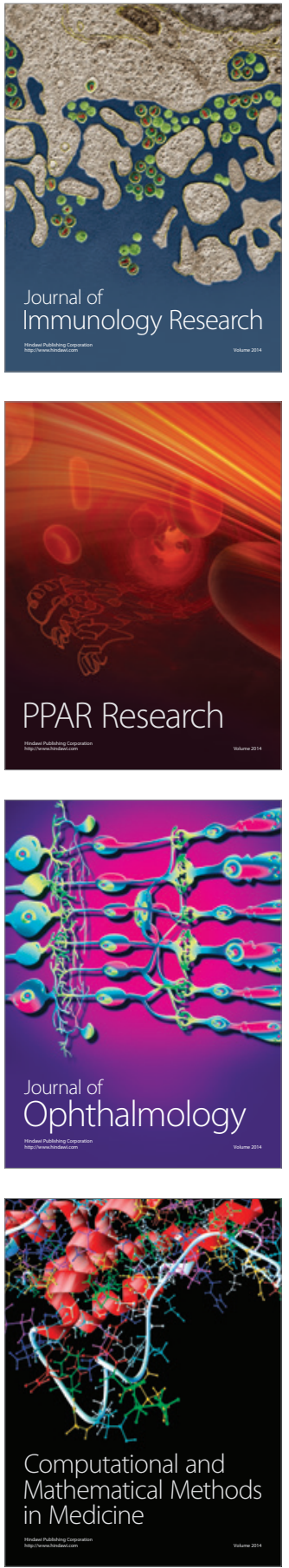

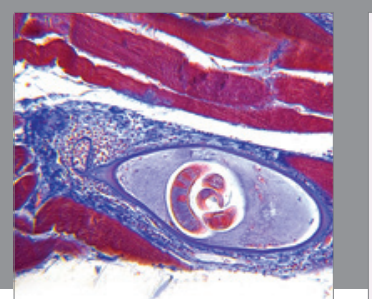

Gastroenterology Research and Practice

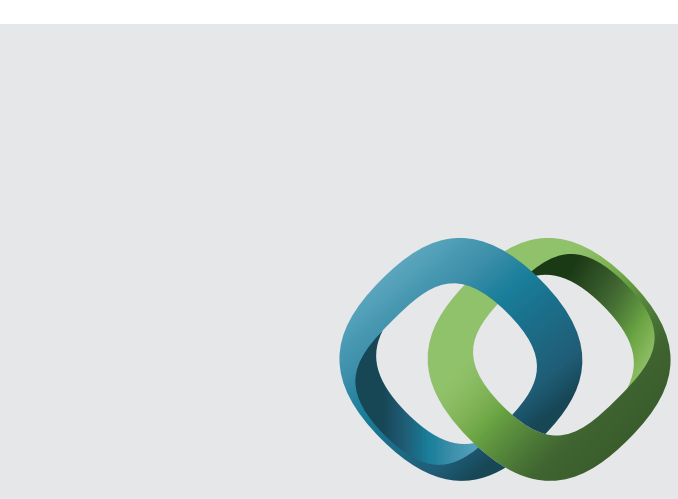

\section{Hindawi}

Submit your manuscripts at

http://www.hindawi.com
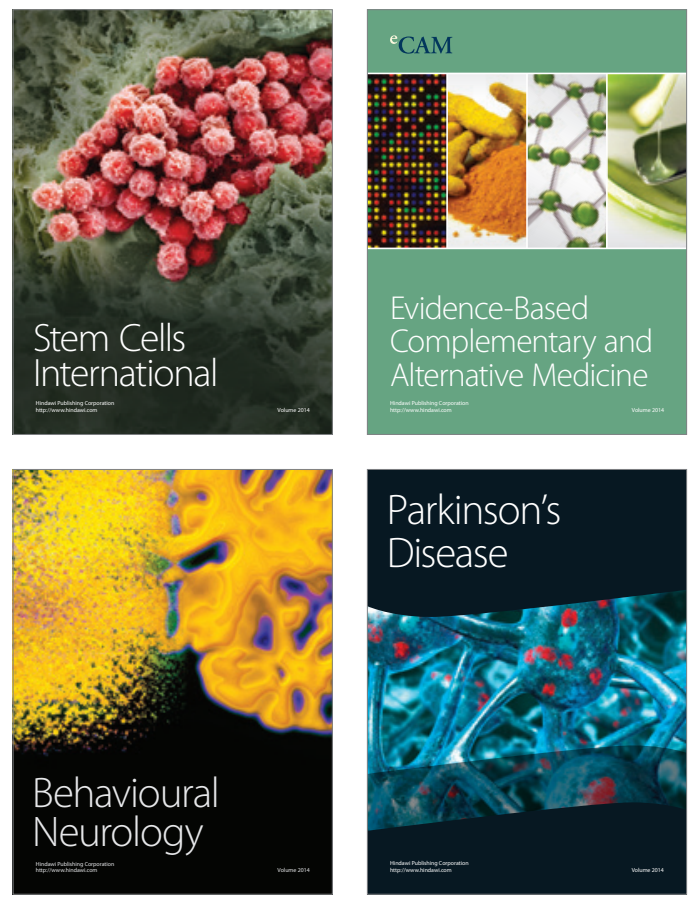
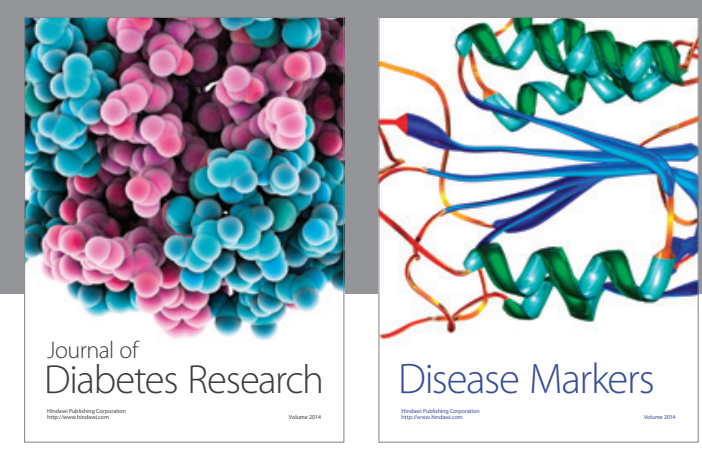

Disease Markers
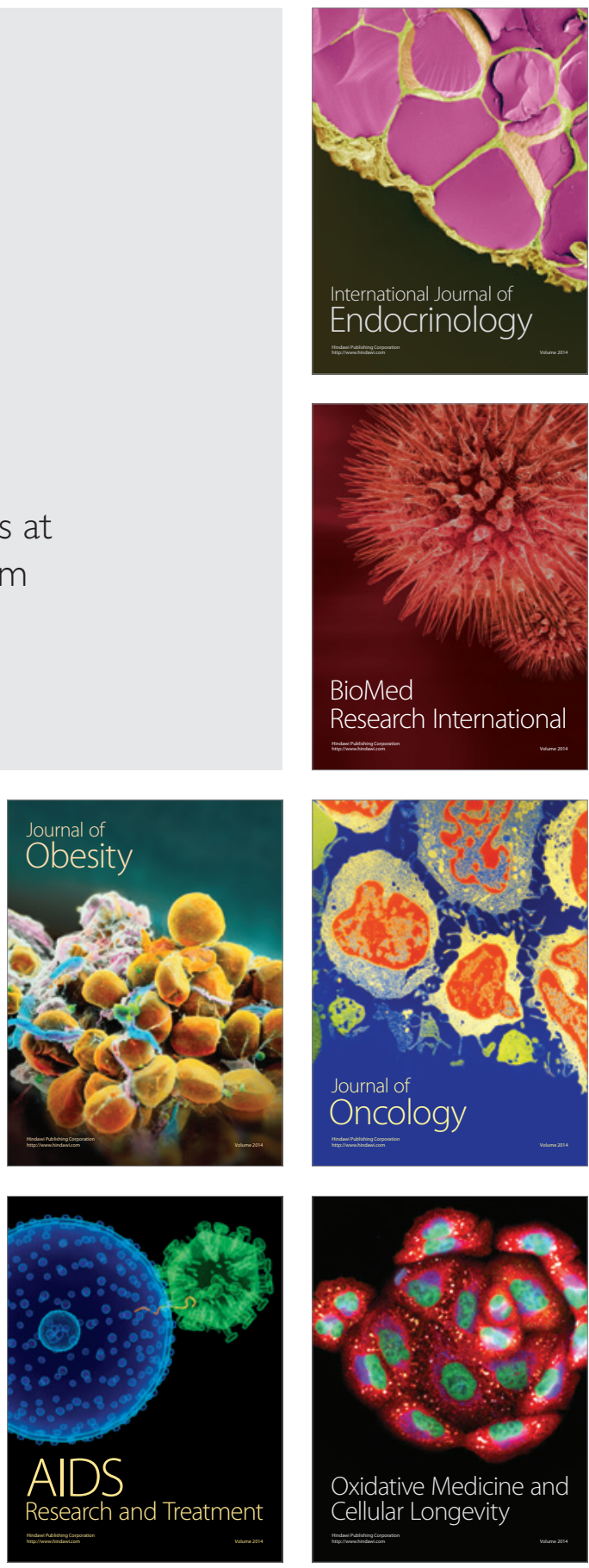